\title{
Nematodes of Astyanax fasciatus (Actinopterygii: Characidae) and their parasitic indices in the Sáo Francisco river, Brazil
}

\author{
Nematoides de Astyanax fasciatus (Actinopterygii: Characidae) e seus índices parasitários do \\ rio São Francisco, Brasil \\ Flavia Guerra Vieira-Menezes ${ }^{1}$; Danielle Priscilla Correia Costa ${ }^{1}$; Marilia Carvalho Brasil-Sato ${ }^{2 *}$ \\ ${ }^{1}$ Curso de Pós-graduação em Ciências Veterinárias, Universidade Federal Rural do Rio de Janeiro - UFRRJ, Seropédica, RJ, Brasil \\ ${ }^{2}$ Departamento de Biologia Animal, Universidade Federal Rural do Rio de Janeiro - UFRRJ, Seropédica, RJ, Brasil
}

Received June 8, 2016

Accepted September 19, 2016

\begin{abstract}
The endoparasite fauna of Astyanax fasciatus from the upper São Francisco river was investigated and ecological parameters and morphological and morphometric data on the parasites are presented. A total of 74 specimens of banded astyanax were collected downstream from the Três Marias dam, municipality of Três Marias, Minas Gerais (18¹2'32"S, $45^{\circ} 15^{\prime} 41^{\prime \prime W}$ ) in January 2011 and January 2012. Eleven taxa of Nematoda were found: Contracaecum sp.; Hysterothylacium sp.; Goezia sp.; Brevimulticaecum sp.; Procamallanus sp.; Procamallanus (Spirocamallanus) saofranciscencis; Cystidicoloides sp.; Spinitectus rodolphiheringi; Rhabdochona sp.; Spiroxys sp.; and Eustrongylides sp.. The fauna of $A$. fasciatus consisted of by larval specimens of Contracaecum sp., Hysterothylacium sp., Brevimulticaecum sp., Cystidicoloides sp., and Spiroxys sp., and by adult specimens of $P$. saofranciscencis, whose prevalence was greater than $10 \%$. Thus, this fish acts as an intermediate host of some species of larval nematodes especially, Anisakidae and Acanthocheilidae (Brevimulticaecum sp., new host record and new locality). It participates in transmitting species such Rhabdochona sp. to carnivorous fish and also acts as a definitive host for $P$. saofranciscencis and S. rodolphiheringi in the upper São Francisco river.
\end{abstract}

Keywords: Anisakidae, Brevimulticaecum sp., endoparasites, nematodes.

\section{Resumo}

A fauna endoparasitária de Astyanax fasciatus do alto rio São Francisco foi investigada e os parâmetros ecológicos e os dados morfométricos e morfológicos dos parasitos apresentados. Um total de 74 espécimes de lambaris foi coletado

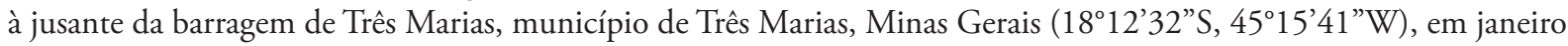
de 2011 e de 2012. Onze táxons de Nematoda foram encontrados: Contracaecum sp., Hysterothylacium sp., Goezia sp., Brevimulticaecum sp., Procamallanus sp., Procamallanus (Spirocamallanus) saofranciscencis, Cystidicoloides sp., Spinitectus rodolphiheringi, Rhabdochona sp., Spiroxys sp. e Eustrongylides sp.. A fauna de A. fasciatus foi caracterizada por espécimes larvais de Contracaecum sp., Hysterothylacium sp., Brevimulticaecum sp., Cystidicoloides sp., Spiroxys sp. e por espécimes adultos de $P$. saofranciscencis, cuja prevalência foi maior que $10 \%$. Assim, o lambari atua como hospedeiro intermediário de algumas espécies de nematoides larvais, especialmente, Anisakidae e Acanthocheilidae (Brevimulticaecum sp., novos hospedeiro e localidade); participa na transmissão de espécies como Rhabdochona sp., para peixes carnívoros, e atua também como hospedeiro definitivo para P. saofranciscencis e S. rodolphiheringi no alto Rio São Francisco.

Palavras-chave: Anisakidae, Brevimulticaecum sp., endoparasitos, nematoides.

\section{Introduction}

Astyanax fasciatus (Cuvier, 1819) is popularly known as the banded astyanax and it is an abundant species in several drainage basins in the Americans (FROESE \& PAULY, 2013). It is classified as a foraging species, and it is well distributed along the Sáo

*Corresponding author: Marilia Carvalho Brasil-Sato. Departamento de Biologia Animal, Universidade Federal Rural do Rio de Janeiro - UFRRJ, Rodovia BR 465, Km 7, CP 74539, CEP 23851-970, Seropédica, RJ, Brasil. e-mail: mcbsato@ufrrj.br
Francisco river (BUCKUP et al., 2007), serving as an important food source for larger fish. Although banded astyanax do not have significant commercial value, they are often eaten as snacks, especially by the riverine population, and they are also used as bait for sport fishing (SANTOS et al., 1995).

Larval specimens of Spiroxys sp. were found in A. fasciatus in Mexico (MORAVEC, 1998). With regard to the same host species on the Nicaraguan coast, Aguirre-Macedo et al. (2001) 
identified larvae of Brevimulticaecum sp., Contracaecum sp. and Spiroxys sp. Subsequently, Salgado-Maldonado (2008) also discovered Procamallanus neocaballeroi (Caballero-Deloya, 1977) and Procamallanus rebecae (Andrade-Salas, Pineda-López, \& García-Magaña, 1994) in Central America.

In the upper São Francisco river, Moreira et al. (1994) recorded occurrences of larvae of Contracaecum sp. and described Procamallanus saofranciscencis (Moreira et al., 1994) in A. fasciatus. Brasil-Sato (2003) further listed occurrences of several species of adult parasitic nematodes in fish in the Sáo Francisco river basin: Cosmoxynema vianai Travassos, 1948; Cosmoxynemoides aguirrei Travassos, 1948; Cucullanus pinnai Travassos, Artigas \& Pereira, 1928; Neocucullanus neocucullanus Travassos, Artigas \& Pereira, 1928; Procamallanus freitasi Moreira, Oliveira \& Costa, 1991; Procamallanus inopinatus Travassos, Artigas \& Pereira, 1928; P. saofranciscencis, Rondonia rondoni Travassos, 1920; Travassosnema travassosi Costa, Moreira \& Oliveira, 1991; Travnema araujoi Fernandes, Campos \& Artigas, 1983; and Travnema travnema Pereira, 1928. Larval specimens of several nematode species were also listed by Brasil-Sato (op. cit.): Contracaecum sp., Cucullanus sp., Dichelyne sp., Eustrongylides sp., Goezia sp., Hysterothylacium sp. and Philometra sp. In addition, Santos et al. (2009) identified larval specimens of Spiroxys sp. in three species of carnivorous fish: Pygocentrus piraya (Cuvier, 1819); Serrasalmus brandtii Lütken, 1875; and Cichla kelberi Kullander \& Ferreira, 2006), in the Três Marias Reservoir. Finally, larval specimens of Rhabdochona sp. were found in Conorhynchos conirostris Valenciennes, 1840, by Brasil-Sato \& Santos (2005).

The aims of this study were to investigate parasitism in A. fasciatus and contribute towards enriching knowledge of the ichthyoparasitology of the São Francisco river. Thus, it had the specific objectives of recording the species of parasitic nematodes of banded astyanax and also measuring their parasitic parameters (prevalence, mean intensity and mean abundance).

\section{Materials and Methods}

A total of 74 specimens of $A$. fasciatus were provided by technical staff of thee regional development company CODEVASF in Três Marias, Minas Gerais, under authorization from the State Forestry Institute of Minas Gerais (IEF-MG). These fish were collected in the upper São Francisco river, located in the municipality of Três Marias, in the state of Minas Gerais (18 ${ }^{\circ} 12^{\prime} 32^{\prime \prime}$ ', $\left.45^{\circ} 15^{\prime} 41^{\prime \prime W}\right)$ in January 2011 and January 2012. The host necropsies and parasite processing procedures followed Eiras et al. (2006). The parasitological descriptors used were those prescribed by Bush et al. (1997). Nematode measurements are presented in millimeters. The measurement ranges are presented in the results tables, and these values are followed by the means and their respective standard deviations. Morphometry was ascertained using a Nikon Alphaphot-2 microscope with a calibrated reticle coupled to the ocular lens. Parasite voucher specimens were deposited in the Helminthological Collection of the Oswaldo Cruz Institute (CHIOC), state of Rio de Janeiro, Brazil. A numbering system was used, as indicated in the results (Table 1). The host voucher specimen was deposited in the Fish of the Museum, University of São Paulo (MZUSP, 95161), state of São Paulo, Brazil.

\section{Results and Discussion}

The parasite fauna of $A$. fasciatus consisted of eleven nematode species, which are presented in Table 1 with their respective parasitic indeces. Nine species were represented by larval specimens and had characterized $A$. fasciatus as a noteworthy intermediate host in their parasitic cycles. Five species (Brevimulticaecum sp., Contracaecum sp., Cystidicoloides sp., Hysterothylacium sp. and Spiroxys sp.) out of these nine in the larval stage presented prevalence higher than $10 \%$. Two nematode species were represented by adult specimens: Spinitectus rodolphiheringi Vaz \& Pereira, 1934 and P. saofranciscencis. For these $A$. fasciatus was characterized as the definitive host. The prevalence of $P$. saofranciscencis was higher than $10 \%$.

The results from the morphometric analysis, i.e. characterization of the peculiarities of nematode species identified in $A$. fasciatus in this study, are shown in Tables 2-5. Exceptionally, the data on Goezia sp. were based on a single specimen $(\mathrm{n}=1 ; \mathrm{P}=1.35 \%)$ that was found in the stomach. Its measurements in millimeters (length $\times$ width) were as follows: body, $1.723 \times 0.105$; esophagus, $0.208 \times 0.025$; ventriculus, $0.028 \times 0.025$; nerve ring from the anterior extremity, 0.108 ; spines of the ventricular appendix region, 0.005; distance between the ring spines of the ventricular appendix region, 0.006; ventricular appendix, 0.743; cecum appendix, 0.088; and tail, 0.058. In the same region of Brazil, Santos-Clapp \& Brasil-Sato (2014) also observed larvae of Goezia sp. in C. kelberi, showing low prevalence (1.8\%). Four species have been described in Brazil: G. spinulosa in Arapaima gigas from Mexiana Island, Amazon river, state of Pará (SANTOS \& MORAVEC, 2009) and from the Araguaia river, state of Mato Grosso do Sul (MENEZES et al., 2011); Astronotus ocellatus, Micropterus salmoides and Pseudoplatystoma corruscans from the Araguaia and Amazon rivers (Pará) (MORAVEC, 1998); G. brasiliensis in the characid Brycon hilarii and in Pseudoplatystoma corruscans from the Paraná river, Foz do Iguaçú and G. brevicaeca in Brycon hilarii from the Paraná river, Foz do Iguaçú (MORAVEC et al., 1994; MORAVEC, 1998); and G. leporini in Leporinus macrocephalus from Batatais, state of São Paulo (MARTINS \& YOSHITOSHI, 2003).

Differing from our study, Santos \& Moravec (2009) and Martins \& Yoshitoshi (2003) found high prevalences of adults and larvae in the species G. spinulosa (90\%) and G. leporini (65\%), respectively. Massive presence of these species gives rise to attacks mainly on the stomach, causing gross lesions and high mortality among the fish.

With regard to $S$. rodolphiheringi, one male, one female, and one juvenile were found in $A$. fasciatus. Their measurements and features were in line with what was reported by Moravec (1998). In the São Francisco river, adult specimens of $S$. rodolphiheringi were found in Franciscodoras marmoratus (Reinhardt, 1874) (SANTOS \& BRASIL-SATO, 2004). Thus, the presence of adult specimens of $S$. rodolphiheringi in $A$. fasciatus increases the list of known hosts in the São Francisco basin.

In this study, the features of $P$. saofranciscencis specimens in $A$. fasciatus, as well as their measurements, were equivalent to what 
Table 1. Prevalence (\%), mean intensity (MI), mean abundance (MA), and their respective standard deviations (SD), followed by amplitude (A), importance of values (IV), and site of infection for nematodes found in Astyanax fasciatus (Cuvier, 1819) of the upper São Francisco river, State of Minas Gerais, Brazil.

\begin{tabular}{|c|c|c|c|c|c|c|}
\hline Nematode species & $\mathbf{P}(\%)$ & $\mathrm{MI} \pm \mathrm{SD}$ & $\mathrm{MA} \pm \mathrm{SD}$ & $\mathbf{A}$ & IV & Site \\
\hline \multicolumn{7}{|l|}{ Larval specimens } \\
\hline \multicolumn{7}{|l|}{ Anisakidae } \\
\hline $\begin{array}{l}\text { Contracaecum sp. } \\
\text { CHIOC n. } 37853\end{array}$ & 58.11 & $5.70 \pm 10.17$ & $3.31 \pm 8.22$ & $1-57$ & Se & AC, E, F, AI, PI \\
\hline Goezia sp. & 1.35 & 1.00 & $0.01 \pm 0.12$ & 1 & Sa & $\mathrm{E}$ \\
\hline \multicolumn{7}{|l|}{ Rhaphydascaridae } \\
\hline $\begin{array}{l}\text { Hysterothylacium sp. } \\
\text { CHIOC n. } 37854\end{array}$ & 59.46 & $4.16 \pm 3.89$ & $2.47 \pm 3.62$ & $1-19$ & Se & $\begin{array}{l}\text { AC, E, F, IC, } \\
\text { AI, PI }\end{array}$ \\
\hline \multicolumn{7}{|l|}{ Acanthocheilidae } \\
\hline $\begin{array}{l}\text { Brevimulticaecum sp. } \\
\text { CHIOC n. } 37851\end{array}$ & 10.81 & $1.88 \pm 2.10$ & $0.20 \pm 0.88$ & $1-7$ & Sa & AC, IC \\
\hline \multicolumn{7}{|l|}{ Camallanidae } \\
\hline $\begin{array}{l}\text { Procamallanus sp. } \\
\text { Cystidicolidae }\end{array}$ & \multicolumn{6}{|c|}{ Cystidicolidae } \\
\hline $\begin{array}{l}\text { Cystidicoloides sp. } \\
\text { CHIOC n. } 37852\end{array}$ & 12.16 & $1.22 \pm 0.44$ & $0.15 \pm 0.43$ & $1-2$ & Sa & $\mathrm{AC}, \mathrm{AI}$ \\
\hline Spinitectus rodolphiheringi* & 1.35 & 1.00 & $0.01 \pm 0.12$ & 1 & Sa & $\mathrm{E}$ \\
\hline \multicolumn{7}{|l|}{ Rhabdochonidae } \\
\hline Rhabdochona sp.** & 5.41 & $8.75 \pm 11.03$ & $0.47 \pm 2.99$ & $1-25$ & Sa & AC, IC, AI \\
\hline \multicolumn{7}{|l|}{ Gnathostomatidae } \\
\hline $\begin{array}{l}\text { Spiroxys sp. } \\
\text { CHIOC n. } 37860\end{array}$ & 22.97 & $3.59 \pm 7.62$ & $0.82 \pm 3.88$ & $1-33$ & Sa & AC, E, F, IC \\
\hline \multicolumn{7}{|l|}{ Dioctophymatidae } \\
\hline Eustrongylides sp. & 1.35 & 1.00 & $0.01 \pm 0.12$ & 1 & Sa & AC \\
\hline \multicolumn{7}{|l|}{ Adult specimens } \\
\hline $\begin{array}{l}\text { Procamallanus saofranciscencis } \\
\text { CHIOC n. } 37857 \text { (male) } \\
\text { CHIOC n. } 37858 \text { (female) }\end{array}$ & 21.62 & $1.06 \pm 0.25$ & $0.23 \pm 0.45$ & $1-2$ & Sa & IC, AI \\
\hline Spinitectus rodolphiheringi & 2.70 & 1.00 & $0.03 \pm 0.16$ & 1 & Sa & $\mathrm{E}$ \\
\hline
\end{tabular}

$\mathrm{Sa}=$ satellite species; $\mathrm{Se}=$ secondary species; $\mathrm{AC}=$ abdominal cavity; $\mathrm{E}=$ stomach $\mathrm{F}=$ liver $\mathrm{IC}=$ intestinal cecum; $\mathrm{AI}=$ anterior intestine; $\mathrm{PI}=$ posterior intestine. Developmental stages: ${ }^{*}$ juvenile; ${ }^{* *}$ larval and juvenile.

was presented by Moravec (1998). Procamallanus saofranciscencis was identified in fish of the families Characidae (VICENTE \& PINTO, 1999) and Acestrorhynchidae (MOREIRA et al., 1994) in the upper Sáo Francisco river. Larval specimens of Procamallanus sp., which had already been identified in this basin, have also been found in Characidae (VICENTE \& PINTO, 1999) and Pimelodidae (BRASIL-SATO \& SANTOS, 2005). Based on the presence of the larval specimens (Procamallanus sp.) and adults of $P$. saofranciscencis in $A$. fasciatus, it can be seen that this foraging fish takes part in the cycle of these species as intermediate and definitive hosts, respectively, and this enlarges the list of hosts for this nematode group in the São Francisco basin.

Larval specimens of Cystidicoloides sp. had already been found in Leporinus friderici (Bloch, 1794) in the upper Paraná river by Takemoto et al. (2009). Thus, $A$. fasciatus is a new host and enlarges the list of the known intermediate hosts of Cystidicoloides sp. in the São Francisco basin.

In this study, larval specimens of Rhabdochona sp. were also found in $A$. fasciatus. They had previously been found in C. conirostris by Brasil-Sato \& Santos (2005). The results obtained in $A$. fasciatus in this study indicate that foraging fish play a role as intermediate hosts for some species of Rhabdochona Railliet, 1916, thus enlarging the list of hosts in the upper São Francisco river.

Larval specimens of Spiroxys sp. were identified in A. fasciatus in Mexico (Moravec, 1998). In the upper São Francisco river, Santos et al. (2009) and Santos-Clapp \& Brasil-Sato (2014) reported occurrences of these larvae in the carnivorous fish Cichla kelberi Kulander \& Ferreira, 2006). In the present study, these larvae were also found in $A$. fasciatus, thus showing that this fish acts as an intermediate host for these parasites.

The occurrences of larval specimens of Hysterothylacium sp., Eustrongylides sp. and Goezia sp. in A. fasciatus enlarges the list of hosts in the São Francisco river, especially given that occurrences of these species had already been identified in several families of fish in the upper São Francisco river (BRASIL-SATO, 2003; SANTOS-CLAPP \& BRASIL-SATO, 2014).

Larvae of Brevimulticaecum sp. were first described in fish by Moravec et al. (1997), but Bruce et al. (1994) asserted that fish rarely act as intermediate hosts for Brevimulticaecum sp. According to Moravec (1998), amphibians are intermediate hosts for Brevimulticaecum spp. and, as Bruce et al. (1994) stated, the most common definitive hosts are reptiles. In the Neotropical region 
Table 2. Morphometry of the larval specimens of the Contracaecum sp., Hysterothylacium sp., and Brevimulticaecum sp. in Astyanax fasciatus (Cuvier, 1819) of the upper Sáo Francisco river, State of Minas Gerais, Brazil.

\begin{tabular}{|c|c|c|c|c|c|c|c|c|c|}
\hline \multirow{2}{*}{$\begin{array}{l}\text { Character of } \\
\text { nematodes }\end{array}$} & \multicolumn{3}{|c|}{$\begin{array}{c}\text { Contracaecum sp. } \\
\text { Larval specimens }(\mathrm{n}=10)\end{array}$} & \multicolumn{3}{|c|}{$\begin{array}{l}\text { Hysterothylacium sp. } \\
\text { Larval specimens }(\mathrm{n}=10)\end{array}$} & \multicolumn{3}{|c|}{$\begin{array}{l}\text { Brevimulticaecum sp. } \\
\text { Larval specimens }(\mathrm{n}=10)\end{array}$} \\
\hline & $\mathrm{X} \pm \mathrm{SD}$ & Min. & Max. & $\mathrm{X} \pm \mathrm{SD}$ & Min. & Max. & $\mathrm{X} \pm \mathrm{SD}$ & Min. & Max. \\
\hline Body $^{L}$ & $3.520 \pm 0.833$ & 2.750 & 4.550 & $1.695 \pm 0.114$ & 1.568 & 1.833 & $3.563 \pm 0.722$ & 2.155 & 4.616 \\
\hline Body ${ }^{w}$ & $0.140 \pm 0.038$ & 0.093 & 0.197 & $0.062 \pm 0.022$ & 0.012 & 0.078 & $0.091 \pm 0.019$ & 0.050 & 0.118 \\
\hline Esophagus $^{\mathrm{L}}$ & $0.259 \pm 0.041$ & 0.201 & 0.309 & $0.167 \pm 0.016$ & 0.143 & 0.183 & $0.442 \pm 0.088$ & 0.303 & 0.550 \\
\hline Esophagus ${ }^{\mathrm{W}}$ & $0.027 \pm 0.013$ & 0.015 & 0.042 & * & * & * & $0.018 \pm 0.002$ & 0.015 & 0.020 \\
\hline Ventriculus $^{\mathrm{L}}$ & $0.055 \pm 0.018$ & 0.041 & 0.083 & $0.026 \pm 0.021$ & 0.015 & 0.064 & $0.046 \pm 0.007$ & 0.035 & 0.053 \\
\hline Ventriculus $^{\mathrm{W}}$ & $0.023 \pm 0.011$ & 0.011 & 0.032 & $0.024 \pm 0.003$ & 0.020 & 0.028 & $0.036 \pm 0.005$ & 0.025 & 0.043 \\
\hline $\begin{array}{l}\text { Ventricular } \\
\text { appendix }\end{array}$ & $0.559 \pm 0.120$ & 0.367 & 0.682 & $0.821 \pm 0.074$ & 0.713 & 0.880 & - & - & - \\
\hline $\begin{array}{l}\text { Ventricular } \\
\text { appendix }^{W}\end{array}$ & * & * & * & * & * & * & - & - & - \\
\hline Nerve ring & $0.182 \pm 0.030$ & 0.144 & 0.220 & $0.115 \pm 0.010$ & 0.105 & 0.125 & $0.124 \pm 0.027$ & 0.090 & 0.153 \\
\hline Excretory & $*$ & * & * & $0.111 \pm 0.009$ & 0.100 & 0.125 & $0.166 \pm 0.014$ & 0.143 & 0.185 \\
\hline $\begin{array}{l}\text { Intestinal } \\
\text { caecum }^{\mathrm{L}}\end{array}$ & $0.314 \pm 0.101$ & 0.227 & 0.482 & $0.027 \pm 0.001$ & 0.025 & 0.028 & $0.215 \pm 0.064$ & 0.113 & 0.370 \\
\hline $\begin{array}{l}\text { Intestinal } \\
\text { caecum }^{\mathrm{W}}\end{array}$ & $*$ & * & * & * & * & * & $0.029 \pm 0.006$ & 0.018 & 0.038 \\
\hline Tail & $0.102 \pm 0.022$ & 0.074 & 0.130 & $0.052 \pm 0.003$ & 0.048 & 0.055 & $0.089 \pm 0.014$ & 0.075 & 0.113 \\
\hline Tooth & $*$ & $*$ & $*$ & $*$ & $*$ & $*$ & $0.004 \pm 0.001$ & 0.003 & 0.004 \\
\hline
\end{tabular}

Table 3. Morphometry of Procamallanus saofranciscencis Moreira, Oliveira \& Costa, 1994 (male and female specimens) and Procamallanus sp. (larval specimens) in Astyanax fasciatus (Cuvier, 1819) of the upper São Francisco river, State of Minas Gerais, Brazil.

\begin{tabular}{|c|c|c|c|c|c|c|c|c|c|}
\hline \multirow{2}{*}{$\begin{array}{l}\text { Character of } \\
\text { nematodes }\end{array}$} & \multicolumn{3}{|c|}{$\begin{array}{c}\text { Procamallanus saofranciscencis } \\
\text { Male specimens }(\mathrm{n}=10)\end{array}$} & \multicolumn{3}{|c|}{$\begin{array}{l}\text { Procamallanus saofranciscencis } \\
\text { Female specimens }(\mathbf{n}=7)\end{array}$} & \multicolumn{3}{|c|}{$\begin{array}{c}\text { Procamallanus sp. } \\
\text { Larval specimens }(\mathrm{n}=3)\end{array}$} \\
\hline & $\mathrm{X} \pm \mathrm{SD}$ & Mín. & Máx. & $\mathrm{X} \pm \mathrm{SD}$ & Mín. & Máx. & sp. 1 & sp. 2 & sp. 3 \\
\hline Body $^{\mathrm{L}}$ & $4.410 \pm 0.454$ & 3.725 & 4.998 & $8.954 \pm 3.132$ & 6.250 & 14.975 & 1.653 & 1.260 & 1.490 \\
\hline Body $^{w}$ & $0.221 \pm 0.033$ & 0.175 & 0.300 & $0.321 \pm 0.057$ & 0.250 & 0.425 & 0.049 & 0.033 & 0.045 \\
\hline $\begin{array}{l}\text { Buccal } \\
\text { Capsule }\end{array}$ & $0.074 \pm 0.008$ & 0.063 & 0.090 & $0.085 \pm 0.026$ & 0.035 & 0.108 & 0.048 & 0.040 & 0.040 \\
\hline $\begin{array}{l}\text { Buccal } \\
\text { Capsule }^{\mathrm{w}}\end{array}$ & $0.074 \pm 0.011$ & 0.063 & 0.084 & $0.123 \pm 0.020$ & 0.103 & 0.155 & 0.033 & 0.150 & 0.015 \\
\hline Basal ring ${ }^{\mathrm{L}}$ & $0.055 \pm 0.008$ & 0.045 & 0.070 & $0.078 \pm 0.007$ & 0.070 & 0.090 & 0.025 & 0.010 & 0.010 \\
\hline Basal ring $^{W}$ & $0.008 \pm 0.001$ & 0.008 & 0.010 & $0.015 \pm 0.001$ & 0.013 & 0.015 & 0.008 & $*$ & 0.013 \\
\hline Spiral number & $13.8 \pm 1.414$ & 12 & 16 & $16 \pm 1.155$ & 14 & 18 & * & - & - \\
\hline $\begin{array}{l}\text { Muscular } \\
\text { esophagus }{ }^{L}\end{array}$ & $0.324 \pm 0.020$ & 0.285 & 0.350 & $0.396 \pm 0.046$ & 0.350 & 0.468 & & & \\
\hline $\begin{array}{l}\text { Muscular } \\
\text { esophagus } w\end{array}$ & $0.112 \pm 0.013$ & 0.098 & 0.140 & $0.139 \pm 0.007$ & 0.133 & 0.150 & $0.200^{\star}$ & $0.138^{\star}$ & $0.125^{\star}$ \\
\hline $\begin{array}{l}\text { Glandular } \\
\text { esophagus }\end{array}$ & $0.502 \pm 0.038$ & 0.452 & 0.570 & $0.569 \pm 0.045$ & 0.530 & 0.638 & & & \\
\hline $\begin{array}{l}\text { Glandular } \\
\text { esophagus }{ }^{W}\end{array}$ & $0.138 \pm 0.020$ & 0.098 & 0.173 & $0.166 \pm 0.013$ & 0.148 & 0.183 & & & \\
\hline Nerve ring ${ }^{A}$ & $0.191 \pm 0.011$ & 0.173 & 0.208 & $0.217 \pm 0.028$ & 0.178 & 0.248 & * & * & * \\
\hline Excretory pore ${ }^{A}$ & $0.258 \pm 0.026$ & 0.228 & 0.310 & $0.313 \pm 0.050$ & 0.243 & 0.390 & * & * & * \\
\hline Spicules $^{\mathrm{La}}$ & $0.103 \pm 0.015$ & 0.080 & 0.113 & - & - & - & * & * & * \\
\hline Spicules ${ }^{\mathrm{S}}$ & $0.084 \pm 0.017$ & 0.063 & 0.671 & & & & $*$ & $*$ & * \\
\hline Vulva $^{B}$ & - & - & - & $3.748 \pm 1.564$ & 2.400 & 6.76 & * & $*$ & * \\
\hline Tail & $0.185 \pm 0.018$ & 0.155 & 0.215 & $0.177 \pm 0.024$ & 0.140 & 0.213 & $*$ & $*$ & * \\
\hline
\end{tabular}

$\mathrm{X}=$ mean; $\mathrm{SD}=$ standard deviation; Min. and Max. = minimum and maximum values for the measurement. Characters: $\mathrm{L}=$ length; $\mathrm{W}=$ width; $\mathrm{A}=$ distance from the anterior end of body; $\mathrm{B}=$ distance from the posterior end of body; La = large; $\mathrm{S}=$ small; -lacks; *not measured; * undifferentiated esophagus. 
(Peru, Venezuela, Nicaragua, Mexico, and Brazil), larvae have been found in the following fish species: A. fasciatus; Gymnotus carapo Linnaeus, 1758; Gymnotus inaequilabiatus (Valenciennes, 1839); Loricariichthys brunneus (Hancock, 1828); Leporinus elongatus Valenciennes, 1850; Leporinus lacustris Amaral Campos, 1945;
L. friderici, Hemisorubim platyrhynchos (Valenciennes, 1840); Hoplias malabaricus (Bloch, 1794); Myleus levis Eigenmann \& McAtee, 1907; Pseudoplatystoma corruscans (Spix \& Agassiz, 1829); Pygocentrus nattereri (Cuvier, 1819); Serrasalmus marginatus (Valenciennes, 1837); Sorubim lima (Bloch \& Schneider, 1801);

Table 4. Morphometry of larval specimens of Cystidicoloides sp., Rhabdochona sp., and Spiroxys sp. in Astyanax fasciatus (Cuvier, 1819) of the upper Sáo Francisco river, State of Minas Gerais, Brazil.

\begin{tabular}{|c|c|c|c|c|c|c|c|c|c|}
\hline \multirow{2}{*}{$\begin{array}{l}\text { Character of } \\
\text { nematodes }\end{array}$} & \multicolumn{3}{|c|}{$\begin{array}{c}\text { Cystidicoloides sp. } \\
\text { Larval specimens }(\mathrm{n}=10)\end{array}$} & \multicolumn{3}{|c|}{$\begin{array}{c}\text { Rhabdochona sp. } \\
\text { Larval specimens }(\mathrm{n}=10)\end{array}$} & \multicolumn{3}{|c|}{$\begin{array}{l}\text { Spiroxys sp. } \\
\text { Larval specimens }(\mathrm{n}=11)\end{array}$} \\
\hline & $\mathrm{X} \pm \mathrm{SD}$ & Mín. & Máx. & $\mathrm{X} \pm \mathrm{SD}$ & Mín. & Máx. & $\mathrm{X} \pm \mathrm{SD}$ & Mín. & Máx. \\
\hline Body $^{\mathrm{L}}$ & $4.615 \pm 0.579$ & 3.785 & 5.704 & $3.409 \pm 1.601$ & 1.646 & 7.164 & $2.145 \pm 0.402$ & 1.725 & 2.871 \\
\hline Body $^{W}$ & $0.104 \pm 0.027$ & 0.056 & 0.127 & $0.078 \pm 0.027$ & 0.044 & 0.127 & $0.056 \pm 0.008$ & 0.043 & 0.069 \\
\hline Vestibule $^{\mathrm{L}}$ & $0.097 \pm 0.015$ & 0.075 & 0.125 & $\star$ & $\star$ & $\star$ & - & - & - \\
\hline Vestibule $^{W}$ & $0.004 \pm 0.001$ & 0.004 & 0.005 & $\star$ & $\star$ & $\star$ & - & - & - \\
\hline Prostom & - & - & - & $0.022 \pm 0.005$ & 0.015 & 0.030 & - & - & - \\
\hline Vestibule & - & - & - & $0.087 \pm 0.012$ & 0.065 & 0.103 & - & - & - \\
\hline \multicolumn{10}{|l|}{ Including prostom } \\
\hline Pseudolabia & $0.008 \pm 0.002$ & 0.005 & 0.010 & - & - & - & $0.019 \pm 0.001$ & 0.018 & 0.021 \\
\hline Deirids & - & - & - & $0.039 \pm 0.006$ & 0.034 & 0.045 & $0.325 \pm 0.030$ & 0.288 & 0.365 \\
\hline $\begin{array}{l}\text { Muscular } \\
\text { esophagus }{ }^{\mathrm{L}}\end{array}$ & $0.214 \pm 0.092$ & 0.148 & 0.380 & $0.218 \pm 0.066$ & 0.138 & 0.323 & $0.115 \pm 0.026$ & 0.080 & 0.150 \\
\hline $\begin{array}{l}\text { Muscular } \\
\text { esophagusw }\end{array}$ & $0.223 \pm 0.006$ & 0.015 & 0.038 & * & * & $*$ & $0.021 \pm 0.004$ & 0.015 & 0.025 \\
\hline Glandular esophagus $^{\mathrm{L}}$ & $1.601 \pm 0.417$ & 1.003 & 2.208 & $1.212 \pm 0.451$ & 0.480 & 2.080 & $0.599 \pm 0.168$ & 0.212 & 0.818 \\
\hline $\begin{array}{l}\text { Glandular } \\
\text { esophagus } \mathrm{w}\end{array}$ & $0.055 \pm 0.020$ & 0.023 & 0.083 & * & * & * & $0.046 \pm 0.007$ & 0.037 & 0.058 \\
\hline Nerve ring ${ }^{A}$ & $0.158 \pm 0.010$ & 0.144 & 0.168 & $0.132 \pm 0.013$ & 0.110 & 0.150 & $0.175^{*}$ & & \\
\hline Excretory pore ${ }^{A}$ & $0.229 \pm 0.054$ & 0.162 & 0.285 & $1.157 \pm 0.040$ & 0.095 & 0.200 & $0.200 \pm 0.016$ & 0.175 & 0.228 \\
\hline Vulva $^{B}$ & - & - & - & & $3.100^{1}$ & & - & - & - \\
\hline Tail & $0.096 \pm 0.014$ & 0.075 & 0.115 & $1.139 \pm 0.035$ & 0.093 & 0.185 & $0.084 \pm 0.014$ & 0.063 & 0.118 \\
\hline
\end{tabular}

Characters: $\mathrm{L}=$ length; $\mathrm{W}=$ width; $\mathrm{A}=$ distance from the anterior end of body; $\mathrm{B}=$ distance from the posterior end of body; -lacks; ${ }^{*}$ not measured; ${ }^{\star}$ measured near prostom; ${ }^{1} \mathrm{n}=1$.

Table 5. Morphometry of Spinitectus rodolphiheringi Vaz \& Pereira, 1934 (male, female, and juvenile specimens) in Astyanax fasciatus (Cuvier, 1819) of the upper São Francisco river, State of Minas Gerais, Brazil.

\begin{tabular}{|c|c|c|c|}
\hline Character of nematodes & $\begin{array}{c}\text { Spinitectus rodolphiheringi } \\
\text { male }(\mathrm{n}=1)\end{array}$ & $\begin{array}{c}\text { Spinitectus rodolphiheringi } \\
\text { female }(\mathrm{n}=1)\end{array}$ & $\begin{array}{c}\text { Spinitectus rodolphiberingi } \\
\text { female juvenile }(\mathrm{n}=1)\end{array}$ \\
\hline Body ${ }^{\mathrm{L}}$ & 2.450 & 4.400 & 1.940 \\
\hline Body ${ }^{w}$ & 0.069 & 0.235 & 0.137 \\
\hline Spine of anterior region & 0.008 & 0.008 & 0.008 \\
\hline Spine of posterior region & 0.004 & 0.004 & 0.004 \\
\hline First ring of spines & 0.043 & 0.081 & 0.053 \\
\hline Vestibule & 0.034 & 0.040 & 0.035 \\
\hline Muscular esophagus ${ }^{\mathrm{L}}$ & 0.150 & 0.235 & 0.110 \\
\hline Muscular esophagusw & * & $*$ & $*$ \\
\hline Glandular esophagus $^{\mathrm{L}}$ & 0.688 & 1.043 & 0.680 \\
\hline Glandular esophagus $^{\mathrm{W}}$ & * & $*$ & $*$ \\
\hline Nerve ring ${ }^{A}$ & 0.158 & $*$ & * \\
\hline Excretory pore $^{A}$ & * & 0.188 & 0.120 \\
\hline Spicules $^{\mathrm{La}}$ & 0.093 & - & - \\
\hline Spicules ${ }^{\mathrm{S}}$ & 0.060 & - & - \\
\hline Vulva $^{B}$ & - & 0.740 & 0.295 \\
\hline Eggs oval $^{\mathrm{L}}$ & - & 0.037 & - \\
\hline Eggs oval ${ }^{\mathrm{w}}$ & - & 0.023 & - \\
\hline Tail & 0.075 & $*$ & 0.060 \\
\hline
\end{tabular}

Characters: $\mathrm{L}=$ length; $\mathrm{W}=$ width; $\mathrm{A}=$ distance from the anterior end of body; $\mathrm{B}=$ distance from the posterior end of body; La = large; $\mathrm{S}=$ small; - lacks; ${ }^{*}$ not measured. 
and Rhamdia guatemalensis (Günther, 1864) (MORAVEC, 1998; AGUIRRE-MACEDO et al., 2001; VIEIRA et al., 2010). Adult specimens of Brevimulticaecum regoi Sprent, 1990, were found in the rays Potamotrygon motoro (Müller \& Henle, 1841) and Potamotrygon castexi Castello \& Yagolkowski, 1969, in southeastern Peru (REYDA, 2008). The present study provides the first record of Brevimulticaecum sp. larvae parasitizing $A$. fasciatus, and this occurrence widens their known geographical distribution in the Neotropical region for the São Francisco river basin. The minimum and maximum values for the measurements on ten larval specimens of Brevimulticaecum sp., followed by their means and standard deviations, are presented in Table 2.

\section{Conclusion}

This study highlights occurrences of and quantitative data on eleven species of parasitic nematodes of $A$. fasciatus in the São Francisco river. The most prevalent species characterizing the nematodes fauna of $A$. fasciatus were, in larval form, Hysterothylacium sp. and Contracaecum sp., followed by Spiroxys sp., Cystidicoloides sp., and Brevimulticaecum sp; and, in adult form, P. saofranciscencis. Except for Contracaecum sp. and $P$. saofranciscencis, $A$. fasciatus represents a new host for these nematode species. These results aid in elucidating the biodiversity of parasites in fish in the Neotropical region; they also add to research projects under development in the São Francisco basin.

\section{Acknowledgements}

The authors wish to thank Dr Yoshimi Sato and Dr Edson Sampaio, researchers at the Hydrobiology and Fish-farming Station of CODEVASF, in Três Marias, Minas Gerais, for their assistance throughout the research process. The authors also wish to acknowledge the CEMIG/CODEVASF agreement that provided appropriate facilities for producing this article. "The first author was granted a fellowship by the Brazilian National Council for Scientific and Technological Development (Conselho Nacional de Desenvolvimento Científico e Tecnológico, CNPq)”.

\section{References}

Aguirre-Macedo ML, Scholz T, Gozález-Solís D, Vidal-Martínez VM, Posel P, Arjona-Torres G, et al. Larval helminths parasitizing freshwater fishes from the Atlantic coast of Nicaragua. Comp Parasitol 2001; 68(1): 42-51.

Brasil-Sato MC. Parasitos de peixes da bacia do São Francisco. In: Godinho HP, Godinho AL. Águas, peixes e pescadores do São Francisco das Minas Gerais. Belo Horizonte: PUC Minas; 2003. p. 149-165.

Brasil-Sato MC, Santos MD. Metazoan parasites of Conorhynchos conirostris (Valenciennes, 1840), an endemic siluriform fish of the São Francisco Basin, Brazil. Rev Bras Parasitol Vet 2005; 14(4): 160-166. PMid:16445873.

Bruce NL, Adlard RD, Cannon LRG. Synoptic checklist of ascaridoid parasites (Nematoda) from fish hosts. Invertebr Taxon 1994; 8(3): 583674. http://dx.doi.org/10.1071/IT9940583.

Buckup PA, Menezes NA, Ghazzi MS. Catálogo das espécies de água doce do Brasil. Rio de Janeiro: Museu Nacional; 2007.
Bush AO, Lafferty KD, Lotz JM, Shostak AW. Parasitology meets ecology on its own terms: Margolis et al. revisited. J Parasitol 1997; 83(4): 575583. PMid:9267395. http://dx.doi.org/10.2307/3284227.

Eiras JC, Takemoto RM, Pavanelli GC. Métodos de estudo e técnicas laboratoriais em parasitologia de peixes. 2. ed. Maringá: Eduem; 2006.

Froese R, Pauly D, editors. FishBase. World Wide Web electronic publication [online]. 2013 [cited 2013 Jan 8]. Available from: http://www.fishbase.org

Martins ML, Yoshitoshi ER. A new nematode species Goezia leporini n. sp. (Anisakidae) from cultured freshwater fish Leporinus macrocephalus (Anostomidae) in Brazil. Braz J Biol 2003; 63(3): 497-506. PMid:14758709. http://dx.doi.org/10.1590/S1519-69842003000300016.

Menezes RC, Santos SMC, Ceccarelli PS, Tavares LER, Tortelly R, Luque JL. Tissue alterations in the pirarucu, Arapaima gigas, infected by Goezia spinulosa (Nematoda). Rev Bras Parasitol Vet 2011; 20(3): 207-209. PMid:21961749. http://dx.doi.org/10.1590/S1984-29612011000300005.

Moravec F, Kohn A, Fernandes BMM. Two new species of the genus Goezia, G. brasiliensis sp. n. and G. brevicaeca sp. n. (Nematoda: Anisakidae), from freshwater fishes in Brazil. Folia Parasitol (Praha) 1994; 41(4): 271-278. PMid:7883259.

Moravec F, Prouza A, Royero R. Some nematodes of frehwater fishes in Venezuela. Folia Parasitol (Praha) 1997; 44(1): 33-47. PMid:9188173.

Moravec F. Nematodes of freshwater fishes of the Neotropical Region. Praha: Academia; 1998.

Moreira NIB, Oliveira CL, Costa HMA. Spirocamallanus inopinatus (Travasso, Artigas \& Pereira, 1928) e Spirocamallanus saofranciscensis sp. n. (Nematoda, Camallanidae) em peixes da represa de Três Marias. Arq Bras Med Vet Zootec 1994; 46(5): 485-500.

Reyda FB. Intestinal helminths of freshwater stingrays in southeastern Peru, and a new genus and two new species of cestode. J Parasitol 2008; 94(3): 684-699. PMid:18605776. http://dx.doi.org/10.1645/GE-1230.1.

Salgado-Maldonado G. Helminth parasites of freshwater fishes from Central America. Zootaxa 2008; 1915: 29-53.

Santos CP, Moravec F. Goezia spinulosa (Nematoda: Raphidascarididae), a pathogenic parasite of the araipama Arapaima gigas (Osteichthyes). Folia Parasitol (Praha) 2009; 56(1): 55-63. PMid:19391331. http://dx.doi. org/10.14411/fp.2009.009.

Santos MD, Albuquerque MC, Monteiro CM, Martins NA, Ederli NB, Brasil-Sato MC. First report of larval Spiroxys sp. (Nematoda, Gnathostomatidae) in three species of carnivorous fish from Três Marias Reservoir, São Francisco River, Brazil. Panam J Aquat Sci 2009; 4(3): 306-311.

Santos MD, Brasil-Sato MC. Parasitos metazoários de Franciscodoras marmoratus (Reinhardt, 1874), "serrudo" (Siluriformes: Doradidae) do rio São Francisco, Brasil. Rev Bras Parasitol Vet 2004; 13(1): 18-22.

Santos RA, Giamas MTD, Campos EC, Camara JJC, Vermulm H Jr. Dinâmica da nutrição do Tambiú Astyanax bimaculatus Linnaeus, 1758 (Pisces, Characiformes, Characidae), na Represa de Ibitinga, Estado de São Paulo, Brasil. Bol Inst Pesca 1995; 22(1): 115-124.

Santos-Clapp MD, Brasil-Sato MC. Parasite community of Cichla kelberi (Perciformes, Cichlidae) in the Três Marias reservoir, Minas Gerais, Brazil. Rev Bras Parasitol Vet 2014; 23(3): 367-374. PMid:25271458. http:// dx.doi.org/10.1590/S1984-29612014059.

Takemoto RM, Pavanelli GC, Lizama MAP, Lacerda ACF, Yamada FH, Moreira LHA, et al. Diversity of parasites of fish from the Upper 
Paraná River floodplain, Brazil. Braz J Biol 2009;69(S2 Suppl): 691-705. PMid:19738975. http://dx.doi.org/10.1590/S1519-69842009000300023.

Vicente JJ, Pinto RM. Nematóides do Brasil. Nematóides de peixes. Atualização: 1985-1998. Rev Bras Zool 1999; 16(3): 561-610. http:// dx.doi.org/10.1590/S0101-81751999000300001.
Vieira KRI, Vicentin W, Paiva F, Pozo CF, Borges FA, Adriano EA, et al. Brevimulticaecum sp. (Nematoda: Heterocheilidae) larvae parasitic in freshwater fish in the Pantanal wetland, Brazil. Vet Parasitol 2010; 172(3-4): 350-354. PMid:20684864. http://dx.doi.org/10.1016/j. vetpar.2010.05.003. 